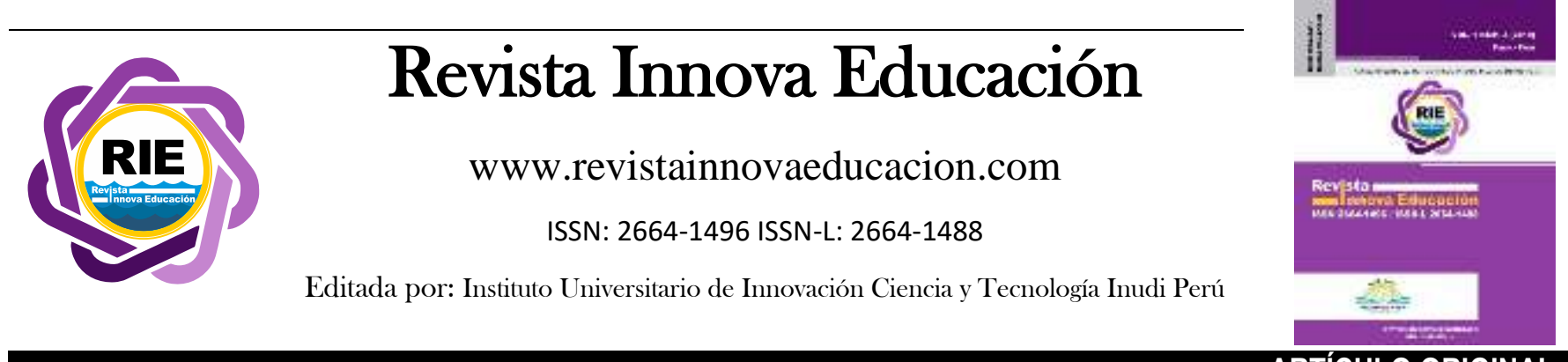

ARTÍCULO ORIGINAL

\title{
Percepción sobre la enseñanza y los aprendizajes en la investigación en una institución de educación superior: hacia la mejora de las capacidades investigativas
}

\section{Perceptions regarding teaching and learning in research at a higher education institution: towards the improvement of research capabilities}

Percepção sobre ensino e aprendizagem em pesquisa em uma instituição de ensino superior: rumo ao aprimoramento das capacidades de pesquisa

\author{
Melissa Rodríguez ${ }^{1}$ \\ Universidad Nacional Autónoma de Honduras, Tegucigalpa - Francisco Morazán, Honduras \\ (iD) https://orcid.org/0000-0003-1772-6097 \\ lourdes.rodriguez@unah.edu.hn (correspondencia)
}

DOI: https://doi.org/10.35622/j.rie.2021.01.010
DOI (Genérico)
: https://doi.org/10.35622/j.rie.2021.01.010
DOI (Documento en español)
: https://doi.org/10.35622/j.rie.2021.01.010.es
DOI (Document in English)
: https://doi.org/10.35622/j.rie.2021.01.010.en

Recibido 24/09/2020/ Aceptado 05/03/2021 Publicado 17/03/2021

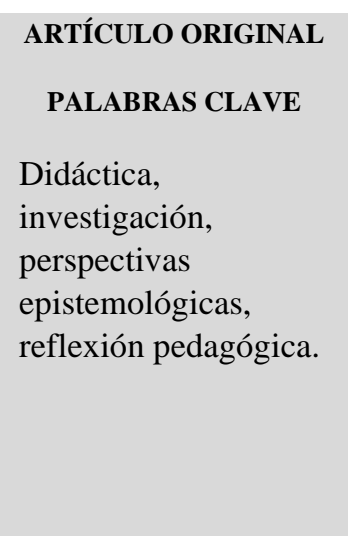

KEYWORDS

Didactics, research, epistemological
RESUMEN. El objetivo de este estudio fue analizar las percepciones de los estudiantes, docentes y autoridades sobre la enseñanza y los aprendizajes en las asignaturas de investigación en la Carrera de Pedagogía y Ciencias de la Educación de la Universidad Nacional Autónoma de Honduras (UNAH), modalidad presencial en Ciudad Universitaria y UNAH-Valle de Sula, I Período Académico 2017. El enfoque fue cualitativo; la población se constituyó con estudiantes matriculados en el Taller de Investigación y con docentes que brindaron las asignaturas investigativas. El muestreo fue intencionado, basándose en criterios de selección, se aplicaron grupos focales y entrevistas semiestructuradas para los docentes. Se concluye que existen debilidades en las prácticas pedagógicas y la necesidad de la reflexión colectiva por parte de los docentes, en consecuencia, en el 2019 se realizó el "Primer Encuentro de Profesores por la Investigación Educativa", estableciendo acuerdos y orientaciones para una Pedagogía de la Investigación.

ABSTRACT. The purpose of this study was to analyze the perception of students, teachers, and authorities about teaching and learning in the research courses of the Pedagogy and Education Sciences Program at the Universidad Nacional Autónoma de Honduras (UNAH for the Spanish acronym), On-campus modality at Ciudad

\footnotetext{
${ }^{1}$ Docente Universidad Nacional Autónoma de Honduras.
} 


\section{perspectives, pedagogic reflection}

\section{PALAVRAS-CHAVE}

Didática, pesquisa, perspectivas epistemológicas, reflexão pedagógica.
Universitaria and UNAH-Valle de Sula, on the first academic term of 2017. The approach of the research was qualitative; the target population was constituted by both, the students enrolled in the research workshop and the teachers who offered research courses. The research presents a Convenience sampling, based on selective criteria, focus groups and semi-structured interviews were applied to the teachers. In conclusion, weaknesses were found in the pedagogical practices and the need for teachers' collective consideration was observed, consequently, in 2019, the "First Gathering of Teachers for the Education Research" took place, establishing agreements and guidelines for a Pedagogy of research.

RESUMO. O objetivo deste estúdio fue analizar as percepções de los estudiantes, docentes y autoridades sobre a enseñanza y los aprendizajes en las assinaturas de investigación en la Carrera de Pedagogía y Ciencias de la Educación de la Universidad Nacional Autónoma de Honduras (UNAH), modalidad presencial en Ciudad Universitaria e UNAH-Valle de Sula, I Período Académico 2017. El enfoque fue cualitativo; la población se constituyó con estudiantes matriculados en el Taller de Investigación y con docentes that brindaron las assignaturas investigativas. El muestreo fue intencionado, basándose en criterios de selección, se aplicaron grupos focales y alterações semiestructuradas para los docentes. Se concluir que existem debilidades nas práticas pedagógicas e necesidad de la reflexión colectiva por parte dos docentes, en consecuencia, en el 2019 se realiza o "Primer Encuentro de Profesores para la Investigación Educativa”, eciendo acuerdos y orientations para una Pedagogía de la Investigación.

\section{INTRODUCCIÓN}

Se investiga con el fin de crear conocimientos que contribuyan a la solución de problemas y a la transformación de la sociedad, por ello se concibe un proceso sistemático y complejo (Latapí, 1994). En las ciencias sociales y/o humanas, el interés radica en el ser humano-cultura y sociedad, y el paradigma de investigación que se utiliza depende de cada ciencia o disciplina en particular. De ahí que los procesos formativos desarrollados en la educación superior deben de ser pertinentes y de calidad. Por otra parte, la enseñanza de la metodología de la investigación conlleva una profunda reflexión (Barriga \& Henríquez, 2004a), no debe de encaminarse a cómo se hace la investigación sino cómo se ha hecho, planteando el desafío de enseñar metodología desde una perspectiva de enseñar investigación. Desde la perspectiva de Rizo (2012) enseñar a investigar está asociado a que el estudiante esté capacitado para construir sus propios objetos de estudios. Canto (2009) y Rojas (2005) consideran que está problemática se relaciona con la percepción que se tiene del contenido, objetivos plasmados en el currículo, la formación del estudiante y lo que interpreta el profesor para su enseñanza.

Lo antes expuesto, da a conocer una problemática recurrente entre qué se debe enseñar ¿metodologías de la investigación o enseñar investigación a nivel de educación superior? como ya lo indican varios autores (Córdoba, 2016). La respuesta a esta pregunta sugiere establecer dos concepciones: la primera referida a los saberes técnicos-conceptuales y la segunda a actitudes para 
el quehacer investigativo. Vinculada a esta problemática están las interpretaciones y creencias del profesor para su enseñanza (Padilla \& Garritz, 2014), así como la actitud y el entusiasmo que debe trasmitir éste a sus estudiantes por la investigación (Aldana, 2012).

Por otra parte, lo que sucede a nivel de pregrado en cuanto a enseñar metodologías o enseñar investigación repercute en lo que acontece en los posgrados como lo señalan las investigaciones de Wainerman (2001) y Fernández \& Wainerman (2015) ya que la finalidad de incentivar la investigación desde la licenciatura es para que los estudiantes decidan seguir formándose en este campo y aporten al desarrollo de conocimientos en las sociedades.

En el contexto de la pedagogía como ciencia que estudia la educación, se plantean diferencias conceptuales, metodológicas y prácticas para entender ¿qué?, ¿cómo? y ¿para qué? se genera el conocimiento en esta ciencia, ya que existen varias concepciones que deben ser reflexionada: a) investigación en educación, b) investigación sobre educación, c) investigación educativa y d) investigación pedagógica referenciados en varios estudios (Restrepo, 1996; Calvo, Camargo, \& Pineda, 2008; Rincón, Murcia, \& López, 2018).

En el marco de estas reflexiones, se infiere la necesidad de establecer claramente cómo construir el saber pedagógico, siendo la enseñanza de la investigación educativa una de las formas idóneas en la formación del profesional de la pedagogía, ya que esta permite la toma de decisiones en los procesos de enseñanza y aprendizaje, en las condiciones y cultura de las instituciones educativas y en los actores involucrados (Piña, 2013), además posee un sustento epistemológico, metodológico y objetivos de investigación específicos con diversidad de enfoques y métodos (López \& Farfán, 2006).

En cuanto a la enseñanza de la investigación educativa, se mantiene una tradición del enfoque cuantitativo para abordar los fenómenos de estudio (Sandín, 2003), sin embargo, existen otras miradas desde lo cualitativo como la investigación - acción educativa (Restrepo, 2004), etnografía de la educación (Maturana \& Garzón, 2015) y ecología del aula (Briones, 2010) que aportarían significativamente en la formación del profesional de la pedagogía.

En este mismo orden de ideas, la bibliografía utilizada representa una problemática para la enseñanza de la investigación educativa, por ser utilizadas como recetas para investigar y con poco fundamento epistemológico. De ahí que una de las reflexiones relevantes sea la comprensión de la epistemología para la construcción del saber científico pedagógico. La epistemología en la formación, corresponde a una evolución histórica de la ciencia y los diferentes postulados 
científicos que separan la metodología de la investigación y epistemología, reflejado en los currículum de enseñanza de las universidades (Cazau, 2011).

Esta problemática en la Carrera de Pedagogía y Ciencias de la Educación de la Universidad Nacional Autónoma de Honduras, es reflejada en antecedentes de investigaciones que consideran que la enseñanza en investigación se vincula con prácticas pedagógicas tradicionales (Ramos, 2015), sin embargo, no existen más registros contextualizados que aborden a profundidad las problemáticas descritas en párrafos anteriores, por eso, surge la interrogante que orientó este estudio ¿cómo es la enseñanza y los aprendizajes en las asignaturas de investigación conforme a las percepciones de estudiantes, docentes y autoridades de la modalidad presencial de la Carrera de Pedagogía y Ciencias de la Educación, Ciudad Universitaria y Valle de Sula en el I período académico 2017 ?

Para concluir, en el estudio se contempló la necesidad de promover espacios para reflexionar en el quehacer docente y la innovación didáctica de la investigación pedagógica (Sánchez, 2004). Para tal efecto, la Carrera de Pedagogía y Ciencias de la Educación a través de la Unidad de Gestión de la Investigación Científica (UGIC-Pedagogía) desarrolló el "Primer Encuentro de Profesores de Pedagogía por la Investigación Educativa" en el III período académico 2019, estableciendo orientaciones prácticas encaminadas a la mejora sustantiva de una pedagogía de la investigación como lo plantea Vega ( 2013).

\section{MÉTODO Y MATERIALES:}

Esta investigación se realizó con el enfoque cualitativo, el cual pretende comprender el fenómeno de estudio desde la intersubjetividad de los actores. Álvarez (2003) indica que la autenticidad de estos estudios radica en que los sujetos puedan expresar su sentir y pensar. El estudio se enmarcó desde una perspectiva fenomenológica, en donde lo cotidiano, lo vivido, y las experiencias de los sujetos conforman estructuras significativas para la comprensión del mundo (Rodríguez et al., 1996).

La población contemplada fue 207 estudiantes inscritos en el primer período académico de 2017 (como se refleja en la tabla 1). Los estudiantes cursaban la asignatura PA- 602, lo que les permitió establecer criterios y valoraciones sobre sus aprendizajes en las asignaturas del área investigativa. 
Tabla 1. Población de estudiantes en Ciudad Universitaria y UNAH-Valle de Sula, I PAC- 2017.

\begin{tabular}{cccc}
\hline \multicolumn{1}{c}{ Asignatura } & $\begin{array}{c}\text { Ciudad } \\
\text { Universitaria }\end{array}$ & $\begin{array}{c}\text { UNAH-Valle de } \\
\text { Sula }\end{array}$ & Total \\
\hline $\begin{array}{l}\text { PA-602 } \\
\text { Taller de Práctica }\end{array}$ & 116 & 91 & 207 \\
\hline \multicolumn{2}{c}{ Fuente: Datos obtenidos del Departamento de Pedagogía CU y Jefatura UNAH -VS }
\end{tabular}

La población docente investigada sumó un total de 86 profesionales que laboran en el Departamentos de Pedagogía de ambas sedes (ver tabla 2.)

Tabla 2. Población docente en Ciudad Universitaria y UNAH-Valle de Sula, I PAC- 2017.

\begin{tabular}{cccc}
\hline Sedes & $\begin{array}{c}\text { Docentes } \\
\text { permanentes }\end{array}$ & Docentes Por hora & Total \\
\hline $\begin{array}{c}\text { Ciudad } \\
\text { Universitaria }\end{array}$ & 34 & 14 & 48 \\
\hline $\begin{array}{c}\text { UNAH-Valle de } \\
\text { Sula }\end{array}$ & 12 & 26 & 38 \\
\hline
\end{tabular}

Fuente: Datos obtenidos del Departamento de Pedagogía CU y Jefatura UNAH -VS

Se utilizó el muestreo tipo intencionado, Galeano (2003) afirma que este corresponde a un perfil de los sujetos que cumplen con criterios de selección propuestos por el investigador con el fin de comprender la realidad de los mismos. Se conformó la muestra con 38 estudiantes cursantes del taller de investigación que contaban con excelencia académica e interés por la investigación, once catedráticos, que impartían las asignaturas investigativas (PA-103 Metodología de la Investigación Educativa I, PA-106 Metodología de la Investigación Educativa II y el PA-602 Taller de Práctica Supervisada II), con experiencia en enseñar dichas asignaturas y cuatro autoridades (Jefe y Coordinador Académico) de las sedes en Ciudad Universitaria y UNAHValle de Sula.

Para el estudio se elaboró una matriz con las siguientes categorías de análisis: perspectivas epistemológicas de los docentes, didáctica de la metodología de la investigación en educación, capacidades investigativas del estudiante y perfil del docente.

Las técnicas que se utilizaron para recabar la información fueron:

a. Grupo focal: Consiste en una entrevista grupal estructurada, con el fin de discutir, compartir 
opiniones, experiencias y percepciones de manera espontánea sobre una guía de preguntas proporcionadas por el moderador (Álvarez, 2003). Fueron organizados seis grupos focales, tres en Ciudad Universitaria (ver figura 1) y tres en UNAH -VS. Para la consulta se desarrolló una guía compuesta por 12 preguntas las cuales surgieron de la matriz de categorización.

\section{b. Entrevista semiestructurada:}

Es un tipo de entrevista, en la que los actores se expresan con libertad en temas propuestos por el entrevistador mediante una guía de preguntas. Esta se desarrolló con los docentes y autoridades seleccionados para el estudio a

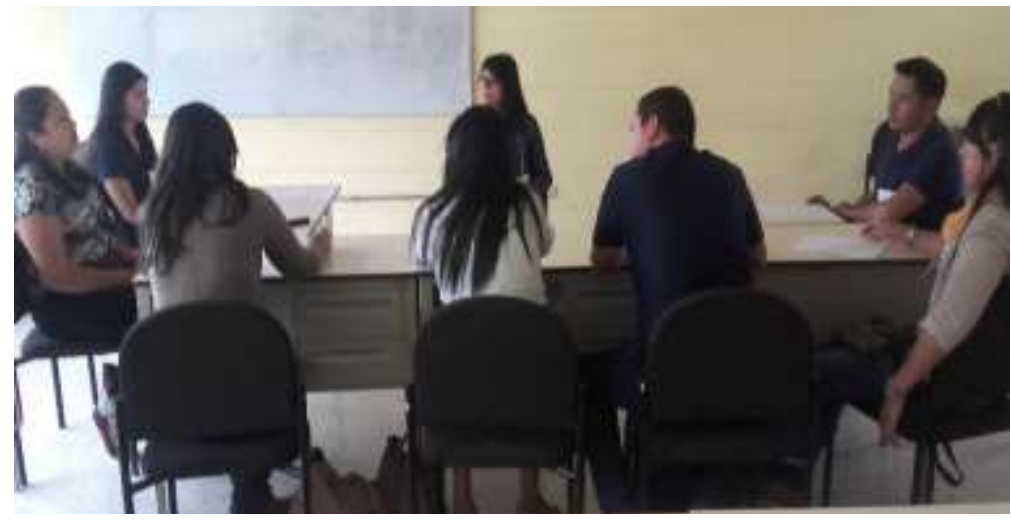

Figura 1. Grupo Focal de Estudiantes en Ciudad Universitaria través de 15 preguntas.

\section{RESULTADOS Y DISCUSIONES}

Para garantizar la confiabilidad y validez de la información se estimó realizar el proceso de análisis de resultados propuesto por Miles y Huberman (1994) citado por Fernández (2006), estableciendo los procesos de reducción de datos, disposición de datos y extracción de conclusiones. Los resultados se estructuran de acuerdo con las categorías de análisis.

\section{Perspectivas epistemológicas de los docentes}

Se concibe como las formas en que el docente entiende el conocimiento y por ende cómo se acerca a comprender la realidad (Barrón, 2015). En esta categoría de análisis se consultó a los docentes sobre ¿qué es enseñar ciencia? Los docentes de Ciudad Universitaria la asocian con la enseñanza de la historia y su abordaje epistemológico, específicamente como la transmisión de información, conceptos, datos, hechos y etapas. En cambio, los docentes de UNAH-Valle de Sula la asocian con la idea apegada a realizar proyectos de investigación. Se infiere que los docentes se enmarcan en un paradigma de la enseñanza de la ciencia asociado al proceso-producto y no al paradigma interpretativo, obstaculizando la posibilidad de promover saberes desde la reflexión y la criticidad para enseñar la ciencia. Son importantes los estudios sobre las creencias y actitudes que poseen los profesores sobre el trabajo que ellos realizan en las aulas (Garritz, 2014). 
Otros de los resultados obtenidos en la investigación, es que todos los docentes opinan que enseñar metodología de la investigación y enseñar investigación son procesos complementarios y complejos. También se logró interpretar que el componente actitudinal hacia la investigación no es reconocido como parte integral de la enseñanza de la investigación. Por otro lado, algunos docentes orientan sus clases a la enseñanza de la investigación, pero desde la mirada procedimental, que conlleva la apropiación de saberes técnico-conceptuales por parte de los estudiantes (Barriga \& Henríquez, 2004b). Con estos resultados, se puede interpretar que las asignaturas del plan de estudio de pedagogía vigente (1994) orientan a la instrumentalización de la metodología de la investigación, limitando la perspectiva de la enseñanza de la investigación educativa en la Carrera.

En cuanto a los paradigmas de investigación educativa; docentes y estudiantes identifican diferencias en la enseñanza de los enfoques metodológicos en las asignaturas de PA-103, PA-106 y PA-602. En UNAH-VS se enfatiza la enseñanza bajo el enfoque cuantitativo, sin embargo, en la asignatura PA-602 las investigaciones son de índole cualitativo y mixto. Resulta oportuno manifestar que se los docentes evidencian cierta conciencia para la enseñanza de los paradigmas de la investigación científica, aunque indican debilidad teórica y metodológica de aplicar dichos enfoques. Basándose en las respuestas de los docentes en UNAH-CU, se considera que el abordaje de los temas educativos corresponde al enfoque cualitativo, no obstante, se identifican debilidades en las capacidades sobre la aplicación de la investigación- acción, como otra mirada cualitativa que acerca al docente a reflexionar sobre su misma práctica (Perines \& Murillo, 2017).

En la problemática anterior, pueden identificarse varias causas entre ellas: la percepción que el docente posee sobre las asignaturas plasmadas en el plan de estudio y la coordinación académica que se realiza en cuanto a la secuencialización de temas, estrategias de enseñanza y de evaluación, y decisiones en cuanto a la enseñanza de enfoques propios de la investigación educativa.

A partir de los resultados, se puede interpretar que no todos los temas investigados en las asignaturas son propiamente del área pedagógica, algunos son de la problemática social y otros son abordados desde las ciencias auxiliares de la pedagogía, como ejemplo se puede citar algunas expresiones de los estudiantes:

"Los embarazos, las incidencias que hay en la adolescencia" (G.F.CU 1: P, 2. E5). "El alcoholismo en los varones" (G.F.VS 3: P, 2. E3). 
El desarrollo de temas de investigación como: el pensamiento, la reflexión y conocimiento del profesor de su práctica pedagógica, el clima organizacional (ecología y etnografía del aula y de la escuela), el profesor como investigador y la pedagogía critica (Briones, 2010) no son considerados como temas de investigación.

Otro hallazgo relevante, es la opinión que posee el estudiante en cuanto a que los docentes en su mayoría no diferencian conceptualmente entre paradigmas, enfoques y métodos de la investigación científica, situación que limita la enseñanza del conocimiento científico. Además de pensar que existe por parte del profesor la preferencia de enseñar desde un enfoque cuantitativo con alcance descriptivo.

Rincón, Murcia y López (2018) apuntan la tendencia de la investigación desde el enfoque cualitativo, persistiendo aún la tendencia positivista de investigar en educación. Por su parte Vélez (2014) indica que la investigación educativa se implementa a partir de la perspectiva epistemológica del investigador, haciendo notar la necesidad de asumir por parte de los docentes y estudiantes una postura reflexiva frente a la perspectiva de los temas investigados y encaminados a la propia realidad educativa hondureña. Las investigaciones pedagógicas deberán recoger los paradigmas socioeducativos desde la teoría crítica de la educación.

Los resultados de esta categoría de análisis, permite entender que las perspectivas epistemológicas del profesorado son congruentes con su quehacer que se traducen en el tipo de formación, experiencia, y desarrollo cultural que poseen, configurando de esa manera un estilo pedagógico (Gimeno, 2003).

\section{Didáctica de la metodología de la investigación en educación}

La segunda categoría de análisis contempló el conocer los objetivos, contenidos y estrategias didácticas y de evaluación que se desarrollan en las asignaturas del área investigativa. Las opiniones reflejaron que los objetivos de enseñanza propuestos por los docentes se encaminan a: 1) explicación del método científico y diseños metodológicos y 2) despertar la motivación e interés en el estudiante por la investigación. Es conveniente señalar que la opinión de los estudiantes sobre los objetivos descritos anteriormente es que estos no son consecutivos en las asignaturas, y que existe prevalencia de la enseñanza de un saber técnico-conceptual.

En cuanto al desarrollo de los contenidos en estas asignaturas, el estudiante expresa que éstos poseen relación directa con la limitada bibliografía utilizada por los docentes; teniendo un 
escaso resultado en el desarrollo de capacidades investigativas en ellos. Para ilustrar lo anterior, se muestra la siguiente opinión:

"Que usen otras bibliografías y que el docente las maneje que está muy acostumbrado a Sampieri y no quiere manejar otras bibliografías (G.F.VS 1: P, 12. E2).

Se identifica que el estudiante es consciente que utilizar una literatura diversa provocaría la visión más amplia de qué y cómo enseñar.

Las estrategias más utilizadas en las clases investigativas fueron: la técnica magistral, el desarrollo de la asesoría individual y grupal, así como el trabajo en equipo; y otras utilizadas con menos frecuencia como el cine-foro (uso de videos educativos), exposiciones por parte de los estudiantes, talleres, guías de trabajo y controles de lectura. Los estudiantes manifestaron que prevalece poca planificación para brindar asesorías por parte del docente y demandan otras estrategias didácticas que involucren el intercambio académico como: profesores invitados, asistencia a seminarios y conferencias de investigación, y comunicación de resultados. Estas expresiones se asocian a una didáctica que debe ser prevista y ejecutada con el fin de alcanzar los objetivos del quehacer de la investigación científica (Sánchez, 2004). Existen otras propuestas metodológicas de cómo investigar utilizando mapas conceptuales, estrategia que el docente puede implementar en el aula (Arellano \& Santoyo, 2012).

Los docentes manifiestan que utilizan en su quehacer una evaluación con función diagnóstica, formativa y sumativa. Desarrolla actividades de socialización de resultados de las investigaciones, pruebas escritas, elaboración de informes finales y artículos científicos, carpetas digitales, uso de la técnica del debate, productos de las diferentes etapas del proceso de investigativo. Además, algunos docentes implementan la coevaluación, autoevaluación y rúbricas. Aunque los estudiantes concuerdan con los docentes, algunos muestran poca aceptación por las estrategias de evaluación, ya que las determinan como tradicionales. Ejemplo de ello es la siguiente opinión:

"Fue como cualquier otra asignatura tres exámenes alguna exposición, la defensa y el informe". (G.F. CU 1: P, 8-E7).

En concordancia con Belmonte (2002) se debe considerar que evaluar no consiste en la calificación de un trabajo final, sino que en una actitud formativa. De ahí la necesidad que el 
docente planifique la evaluación de los aprendizajes, comunique a los estudiantes sobre los criterios de calidad que serán aplicados en las asignaturas; y valore la retroalimentación como un principio para la mejora continua.

Los estudiantes opinan que los docentes no están totalmente desorientados en cómo enseñar; sin embargo, la visión convencional y lineal sigue predominando en las planificaciones didácticas haciendo uso de procedimientos, medios, recursos y herramientas limitados. Se deduce de ello, la importancia de reflexionar sobre la apropiación de una didáctica de la investigación que considere el modelo práctico del aprendizaje.

Consciente de esta problemática y en procura de una innovación didáctica en el aula de clases, el trabajo docente de ser fortalecido con una actitud y disposición hacia el cambio. Se podría innovar considerando otras propuestas teóricas metodológicas que contribuyan a que los estudiantes aprendan sobre investigación y a investigar (Morales et al., 2005). Asimismo, reflexionar sobre la relación entre estilos de aprendizaje y estilos de enseñanza para comprender aún más las prácticas pedagógicas (Ventura, 2016).

\section{Capacidades investigativas del estudiante}

Esta categoría de análisis aborda las destrezas, habilidades y actitudes desarrolladas por los estudiantes en las asignaturas del área investigativa. Los docentes consideran que las habilidades y destrezas que poseen los estudiantes son: formular el planteamiento de investigación, diseñar una propuesta de intervención; y elaborar artículos científicos; también expresaron que existen debilidades en los estudiantes en cuanto a identificar el fenómeno de estudio, carencia de una lógica investigativa y problemas a confundir la metodología del enfoque cuantitativo y cualitativo. En cambio, los estudiantes manifiestan que sus habilidades están dirigidas a la búsqueda de información confiable, elaboración de hipótesis y objetivos, manejo de técnicas para la recolección de datos, la aplicación de normas APA, interpretación de los resultados de forma reflexiva, habilidades tecnológicas en el manejo del software SPSS (Statistical Package Social Science), igualmente otras habilidades expresadas se orientan a la búsqueda en repositorios como la Biblioteca Virtual de la Universidad y el uso del gestores bibliográficos como CITAVI.

De acuerdo con los argumentos de los estudiantes, las actitudes que ellos han desarrollado en las asignaturas del campo investigativo son de carácter genérico, a la vez, que mencionan la responsabilidad, trabajo colaborativo y autónomo, compromiso, dedicación; en oposición al poco desarrollo de una actitud hacia la investigación propiamente. Estas consideraciones reflejan aún 
más la prevalencia de una enseñanza desde la metodología y técnica de la investigación. Un ejemplo de dichas opiniones es la siguiente:

\section{"Investigar cualquier problema sencillo que ocurra en su entorno".}

\section{(G.F. CU 1: P, 7. E6).}

A partir de los resultados obtenidos queda reflejado el vínculo entre la disposición personal hacia la investigación y la motivación e interés que despierta el docente en los estudiantes. Los estudiantes valoran que sus docentes enseñen haciendo uso de sus investigaciones como recursos didácticos. La actitud hacia la investigación es generada por la confianza académica y experiencia en investigación que inspiran los docentes (Rojas et al., 2012), aunado a eficientes procesos de asesoraría y acompañamiento de las prácticas investigativas de los estudiantes. Posee un gran valor el concepto de autoeficacia y el interés para el aprendizaje de la investigación en los estudiantes, ya que es asociado al recorrido curricular y a las visiones que son proporcionadas por el colectivo de docentes (Criollo et al., 2017).

\section{Perfil del docente}

En la última categoría de análisis, los resultados conllevan a que en la enseñanza de la metodología, el docente es un expositor de teorías, métodos, técnicas e instrumentos de investigación para orientar la producción de conocimientos acerca de fenómenos educativos, contrariamente a la enseñanza de la investigación en la que el docente pretende desarrollar actitudes hacia la investigación (incentivar al estudiante para cuestionar lo que conoce, formular preguntas, encontrar respuestas), así como desarrollar habilidades para la construcción de objetos de estudios propios. En ese sentido, la enseñanza de las asignaturas investigativas el docente debería ser asesor y orientador que acompaña al estudiante en su formación (Acosta, 2014).

Los docentes reconocen que poseen habilidades y destrezas para la construcción del objeto de estudio, manejo de software estadísticos y cualitativos, dominio de las normas APA, experiencia en investigaciones educativas y pedagógicas. También opinan que cuentan con actitudes como el compromiso y dedicación por sus estudiantes, disposición hacia la formación continua en investigación, y poseer interés por la investigación como parte del quehacer del docente universitario. Esto afianza aún más la idea de acercar la investigación a la docencia (Tesouro \& Puiggalí, 2014). Sin embargo, se evidencia debilidades para alcanzar un perfil idóneo, como lo refleja la siguiente opinión. 
"La Universidad no ha generado espacios realmente activos de formación permanente en el ámbito de investigación" (Entrevista 5: P, 13. D-VS).

Además de lo anterior, se debe valorar otras fortalezas del profesorado como: la experiencia de su labor en el sistema educativo nacional, su formación de posgrado en universidades nacionales e internacionales y la apertura a la actualización y capacitación en temas del área investigativa. Esto se refuerza en la postura de Aldana \& Joya (2011) al expresar que el docente debe incursionar constantemente en el quehacer investigativo de esa manera influirá en el estudiante una actitud hacia la investigación. En contraste, los estudiantes manifestaron que sus profesores no demuestran en las clases experiencia en el campo investigativo.

Finalmente, se puede enunciar otros factores que contribuyen a comprender la escasa preparación que poseen los docentes respecto a la capacitación sobre paradigmas de la investigación y abordajes epistemológicos del campo de la educación, así como mejorar las condiciones para desarrollar las asignaturas. Esta última en referencia, a las demandas que los mismos docentes manifiestan al no contar con espacios físicos (laboratorio de computación), recursos (bibliografía especializada y diversa sobre investigación educativa) y la asignación de la carga académica de acuerdo con su área de formación. La siguiente opinión refleja lo expresado anteriormente:

"Si queremos mejorar la calidad de la educación hay que ubicar a cada docente de acuerdo con su formación y su competencia..."

(Entrevista 3: P, 12. A-CU).

\section{CONCLUSIONES}

Las prácticas pedagógicas desarrolladas por los docentes en las asignaturas de investigación en la Carrera de Pedagogía y Ciencias de la Educación se ubican en una visión del paradigma racional técnico, considerándose no apto para la enseñanza de la investigación; de igual manera, estas prácticas de los docentes corresponden a una perspectiva epistemológica que se relaciona con las creencias que él mismo posee sobre cómo enseñar ciencia.

Resulta una prioridad generar procesos de reflexión colectivos en la Carrera de Pedagogía tomando en cuenta ambas sedes (Ciudad Universitaria y Valle de Sula), con el propósito de debatir en cuanto a enseñar metodología de la investigación y enseñar a investigar; en el estudio realizado se apreció que no existen consensos por los actores en cuáles son las capacidades investigativas 
que se pretenden formar en el profesional de la Pedagogía. Se demuestra una fuerte tendencia hacia la enseñanza del enfoque cuantitativo, descuidando, la formación en el enfoque cualitativo para abordar los fenómenos propios del campo pedagógico. En consecuencia, esta situación provoca que no se desarrollen en las asignaturas investigaciones desde miradas cualitativas como la fenomenología, etnografía, investigación acción, teoría fundamenta, etnometodología entre otros métodos. En este mismo orden de ideas, se reconoce la falta capacitación y/o actualización de estos enfoques por parte de los docentes. Ante esta situación planteada, se limita una formación consolidada en el saber pedagógico para los estudiantes de la Carrera.

Para el desarrollo de la investigación como área de formación del pedagogo, es necesario que las asignaturas de este campo no sean asignadas a docentes que no poseen ningún interés y/o no estén comprometidos con el quehacer investigativo; adicional a esto, es urgente una gestión administrativa eficiente, así como una coordinación del trabajo entre los docentes que imparten las asignaturas. Otras de las grandes debilidades ilustradas a partir de las opiniones de los actores son los pocos recursos para el desarrollo de las asignaturas como el no contar con espacios físicos idóneos como salas de cómputo bien equipadas con software especializados en investigación y medios de divulgación como una revista científica propia de la disciplina y adscrita al Departamento de Pedagogía; y por último la falta de bibliografía especializada y actualizada sobre investigación educativa.

En consecuencia, se debe replantear el perfil del pedagogo en el plan de estudio con el fin de fortalecer las competencias investigativas en el futuro profesional quien se desempeñará en contextos laborales socioeducativos cambiantes.

Se deben generar los espacios de reflexión permanentes en la Carrera de Pedagogía, con el propósito que el profesorado reconozca las debilidades y fortalezas sobre sus prácticas pedagógicas en la enseñanza de las asignaturas de investigación; así como proponer soluciones conjuntas con otros actores involucrados en el proceso formativo, a la vez se debe establecer una coordinación técnica-metodológica entre docentes para atender las asignaturas de esta área. Se demanda procesos de capacitación y/o grupos de discusión instaurados con el fin de reforzar los paradigmas de la investigación en educación. Por estas razones antes descritas, la Unidad de Gestión de la Investigación Científica de Pedagogía (UGIC-Pedagogía) instituida en el 2016 es la responsable de asumir esta función trascendental de coordinar procesos de cambio e innovación en la Carrera. 
Se debe priorizar una gestión eficiente para la adquisición de medios y recursos (espacios tecnológicos, acervo bibliográfico actualizado, medios de divulgación científica) por parte de las autoridades de la Carrera de Pedagogía, a fin de atender las demandas y necesidades de los estudiantes y docentes, procurando constituir procedimientos para la capacitación y/o actualización de los docentes.

\section{Hacia la mejora de las capacidades investigativas en la Licenciatura de Pedagogía y Ciencias de la Educación.}

Como extensión y puesta en práctica del desarrollo del estudio, la Unidad de Gestión de la Investigación Científica de Pedagogía, propuso, planificó y llevó a cabo los días 29 y 30 de agosto de 2019, el Primer Encuentro de Profesores de Pedagogía por la Investigación Educativa, mediante el cual se estableció la ruta "hacia la mejora de las capacidades investigativas en la licenciatura de Pedagogía”, considerando al profesorado de la Carrera en las modalidades presencial y a distancia, los cuales fueron

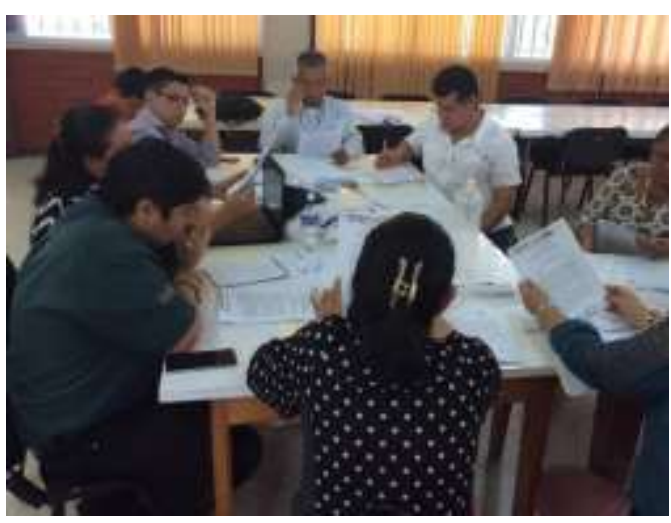

Mesa de trabajo Fundamentos Epistemológicos de la investigación participes del proceso de reflexión convocado.

Se estableció como objetivo esencial el reflexionar sobre la práctica docente en la enseñanza de la investigación en la Carrera de Pedagogía, permitiendo el compartir experiencias, fortaleciendo conocimientos, centrando la discusión en el mejoramiento de las capacidades investigativas en los estudiantes. La metodología del encuentro fue organizada en dos etapas, la primera que consistió en el desarrollo de conferencias y un taller sobre investigación cualitativa por parte de un especialista en educación de Costa Rica, la segunda etapa se llevó a cabo en tres procesos, una ponencia presentada los resultados de la investigación objeto de este artículo, mesas de trabajo y un plenario general.

A continuación, se presenta la figura 2 en la que se esbozan los ejes problematizadores y algunos de los resultados que surgieron del debate y discusión por parte de los docentes.

La figura 2, presenta cuatro ejes que orientaron el evento académico: a) Fundamentos epistemológicos de la investigación b) La educación como fenómeno social c) La investigación educativa desde el enfoque cualitativo d) La formación del profesor universitario en el área 
disciplinar y pedagógica. Se establecieron algunas reflexiones como la necesidad de construir el saber científico de la pedagogía determinando para ello, la necesidad de desarrollar el discurso pedagógico nacional. Por otra parte, se deben de consolidar procesos de formación pertinentes y construir una cultura investigativa en la Carrera. En cuanto a formación del profesorado universitario en el área disciplinar y pedagógica, los docentes manifestaron la necesidad de incentivos como pasantía, intercambios a nivel nacional e internacional, la necesidad de ampliar la oferta en formación de investigadores, promover convenios con organismos internacionales, desarrollo de eventos académicos de calidad, entre otros.

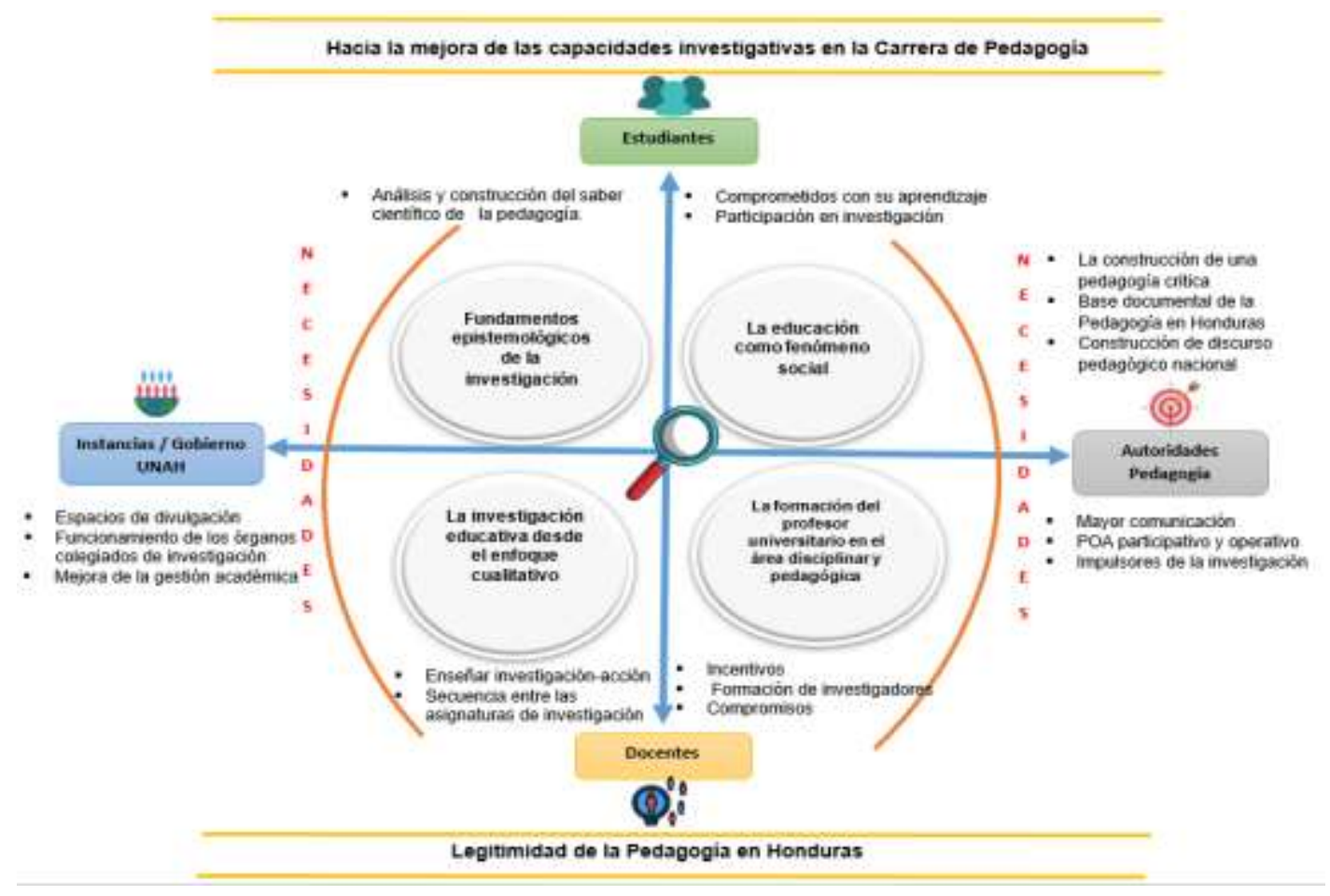

Figura 2. Ejes problematizadores y sus resultados

Fuente: Elaboración Propia.

Los docentes universitarios deben de empoderarse de la función esencial de la investigación en la universidad, de tal manera los académicos sostienen que el conocimiento epistemológico de la ciencia tiene que ser una de las competencias del perfil profesional del profesor universitario, su papel es formar profesionales universitarios con pensamiento científico, crítico y reflexivo. Finalmente, se demanda la toma de decisiones pertinentes y una planificación prospectiva por parte de las autoridades de la Carrera y de la UNAH con el fin de generar cambios en la formación y desarrollo de la investigación. 
Agradecimientos / Acknowledgments:

Expreso mi agradecimiento a los docentes del Departamento de Pedagogía y Ciencias de la Educación de la Facultad de educación de la Universidad Nacional Autónoma de Honduras.

Conflicto de intereses / Competing interests:

El autor declara que no incurre en conflictos de intereses.

Rol de los autores / Authors Roles:

No aplica.

Fuentes de financiamiento / Funding:

El autor declara que no recibió un fondo específico para esta investigación.

Aspectos éticos / legales; Ethics / legals:

El autor declara no haber incurrido en aspectos antiéticos, ni haber omitido aspectos legales en la realización de la investigación.

\section{REFERENCIAS BIBLIOGRÁFICAS}

Acosta, S. (2014). La investigación en el aula. Aprender a conocer. Trillas.

Aldana, G. (2012). La formación investigativa: su pertinencia en pregrado. Revista Virtual Universidad Católica Del Norte, 36, 379. http://revistavirtual.ucn.edu.co/index.php/RevistaUCN/article/view/366

Aldana, G., \& Joya, N. (2011). Actitudes hacia la investigación científica en docentes de metodología de la investigación. Tabula Rasa, 14, 295-309. https://doi.org/10.25058/20112742.428

Álvarez, J. (2003). Cómo hacer investigación cualitativa. Fundamentos y metodología. Paidós Educador.

Arellano, J., \& Santoyo, M. (2012). Investigar con mapas conceptuales. Narcea.

Barriga, O., \& Henríquez, G. (2004a). Artesanía y técnica en la enseñanza de la metodología de la investigación social. Cinta de Moebio: Revista Electrónica de Epistemología de Ciencias Sociales, 126-131. https://dialnet.unirioja.es/servlet/articulo?codigo=1065040

Barriga, O., \& Henríquez, G. (2004b). Revalorando lo artesanal. Lo sustratos de los objetivos pedagógicos en la enseñanza de la metodología de la investigación social. Investigaciones Sociales, VIII(13), 311-324. https://doi.org/https://doi.org/10.15381/is.v8i13.6933

Barrón, C. (2015). Concepciones epistemológicas y práctica docente. Una revisión. REDU: Revista de

Docencia

Universitaria, $13(1)$,

http://dialnet.unirioja.es/servlet/articulo?codigo=5027835\&info=resumen\&idioma=ENG

Belmonte, M. (2002). Eneñar a investigar. Orientaciones prácticas. Ediciones Mensajero. 
Briones, G. (2010). Tendencias recientes de la investigación en pedagogía. Áreas, Problemas y Formas de Relación. Centros de Estudios de Opinión, 1-23.

Calvo, G., Camargo, M., \& Pineda, C. (2008). ¿Investigación educativa o investigación pedagógica? El caso de la investigación. MAGIS. Revista Internacional de Investigación En Educación, 1(1), 164-174. http://www.redalyc.org/articulo.oa?id=281021687011

Canto, J. (2009). Metodología de la investigación en el nivel superior. Cuestiones epistemológicas en su enseñanza. Metodología de La Ciencia. Revista de La Asosiación Mexicana de Metodología de La Ciencia y de La Investigación, 1(1), 142-163. http://www.ammci.org.mx/revista/pdf/Seccion metodologia de la ciencia (2a parte)/MetInvESCanto.pdf

Cazau, P. (2011). Evolución de las relaciones entre la epistemología y la metodología de la investigación.

Paradigmas, $3(2)$,

$109-126$. https://dialnet.unirioja.es/servlet/articulo?codigo=3798214

Córdoba, M. (2016). Reflexión sobre la formación investigativa de los estudiantes de pregrado. Revista Virtual Universidad Católica Del Norte, 47, 20-37. https://www.redalyc.org/pdf/1942/194244221003.pdf

Criollo, M., Romero, M., \& Fontaines, T. (2017). Autoeficacia para el aprendizaje de la investigación en estudiantes universitarios. Psicologia Educativa, 23(1), 63-72. https://doi.org/10.1016/j.pse.2016.09.002

Fernández, L. (2006). ¿Cómo analizar datos cualitativos? Butlletí La Recerca, 1-13.

Fernández, L., \& Wainerman, C. (2015). La dirección de tesis de doctorado: ¿una práctica pedagógica? Perfiles Educativos, XXXVII(148), 156-171. http://www.scielo.org.mx/pdf/peredu/v37n148/v37n148a10.pdf

Galeano, M. (2003). Diseño de proyectos en la investigación cualitativa. Fondo Editorial Universidad EAFIT.

Garritz, A. (2014). Creencias de los profesores, su importancia y cómo obtenerlas. Educación Química, 25(2), 88-92. https://doi.org/10.1016/S0187-893X(14)70529-4

Gimeno, J. (2003). El alumno como invención. Morata.

Latapí, P. (1994). Reflexiones acerca del "éxito" de la investigación educativa. In La investigación 
educativa en México (p. 243). Fondo de Cultura Económica.

López, A., \& Farfán. (2006). Congreso Estatal de Investigación Educativa. Actualidad, Prospectivas y Retos. Congreso Estatal de Investigación Educativa, 9. https://atenea.epn.edu.ec/bitstream/25000/344/1/La-investigación-educativa-como-base-dela-nueva-educación-LOPEZ-Y-FARFAN.pdf

Maturana, G., \& Garzón, C. (2015). La etnografía en el ámbito educativo. Educación y Desarrollo Social, 9(2), 192-205. https://doi.org/https://doi.org/10.18359/reds.954

Morales, O., Rincón, Á., \& Tona, J. (2005). Cómo enseñar a investigar. Educere, 9(1316), $217-$ 224. http://redalyc.uaemex.mx/redalyc/src/inicio/ArtPdfRed.jsp?iCve=35602910

Padilla, K., \& Garritz, A. (2014). Creencias epistemológicas de dos profesores-investigadores de la educación superior. Educación Química, 25(3), 343-353. https://doi.org/https://doi.org/10.1016/S0187-893X(14)70550-6

Perines, H., \& Murillo, F. (2017). ¿Cómo mejorar la investigación educativa? Sugerencias de los docentes. Revista de La Educacion Superior, 46(181), 89-104. https://doi.org/10.1016/j.resu.2016.11.003

Piña, J. (2013). Investigación educativa ¿para qué? Perfiles Educativos, XXXV(139), 3-6. https://www.redalyc.org/pdf/132/13225611010.pdf

Ramos, S. (2015). La Enseñanza de la Investigación Científica en la Universidad Nacional Autónoma de Honduras Valle de Sula, UNAH-VS. [Universidad Nacional Autónoma de Honduras]. https://tzibalnaah.unah.edu.hn/bitstream/handle/123456789/5958/TMSies00036.pdf?sequence=2\&isAllowed=y

Restrepo, B. (1996). Investigación en Educación. Instituto Colombiano para el Fomento de la Educación Superior.

Restrepo, B. (2004). La investigación-acción educativa y la construcción de saber pedagógico. Educación y Educadores, 7, 45-55. http://www.redalyc.org/articulo.oa?id=83400706

Rincón, L., Murcia, N., \& López, C. (2018). Perspectivas de investigación en educación y pedagogía. El Ágora USB, 18(1), 73. https://doi.org/10.21500/16578031.3212

Rizo, M. (2012). Enseñar a investigar investigando. Experiencias de investigación en comunicación con estudiantes de la Licenciatura en Comunicación y Cultura de. 20-39. 
Rodríguez, G., Gil, J., \& García, E. (1996). Capítulo II. Métodos de Investigación Cualitativa. In Metodología de la Investigación Cualitativa (p. 378). ALJIBLE.

Rojas, H., Méndez, R., \& Rodríguez, Á. (2012). Índice de actitud hacia la investigación en estudiantes del nivel de prgrado. Entramado, 17(2), 216-229. http://www.scielo.org.co/scielo.php?pid=S190038032012000200015\&script=sci_abstract\&tlng=es

Rojas, R. (2005). Proceso de enseñanza -aprendizaje de la metodología de invetigación. In Formación de Investigadores Educativos. Una propueta de Investigación. (Décima pri, p. 252). Plaza y Valdes.

Sánchez, R. (2004). Enseñar a investigar. Una didáctica nueva de la investigación en ciencias sociales y humanas (Segunda). Instituto de Investigaciones sobre la Universidad y la Educación.

Sandín, M. (2003). Investigación Cualitativa en Educación Fundamentos y Tradiciones. In Mcgraw Hill.

Tesouro, M., \& Puiggalí, J. (2014). La relación entre la docencia y la investigación según la opinión del profesorado universitario. Procedia - Social and Behavioral Sciences, 196, 212218. https://doi.org/10.1016/j.sbspro.2015.07.031

Vega, D. (2013). Pedagogía de la Investigación: Problematización de la Enseñanza-Aprendizaje. $\begin{array}{lllll}\text { Educcación } & y & \text { Territorio, } & 3(1), & 155-172 .\end{array}$ https://www.jdc.edu.co/revistas/index.php/reyte/article/view/399

Vélez, D. (2014). Perspectivas epistemológicas para la investigación educativa: aspectos fundametnales, teóricos y metodológicos. Grupo Editorial Éxodo.

Ventura, A. (2016). ¿Enseño como aprendí?: el rol del estilo de aprendizaje en la enseñanza del $\begin{array}{llll}\text { profesorado } & \text { universitario. Aula Abierta, 44(2), 98. }\end{array}$ https://doi.org/10.1016/j.aula.2016.05.001

Wainerman, C. (2001). Acerca de la formación de investigadores en ciencias sociales. In $\mathrm{La}$ Trastienda de la Investigación (pp. 19-26). Ediciones Lumiere. 